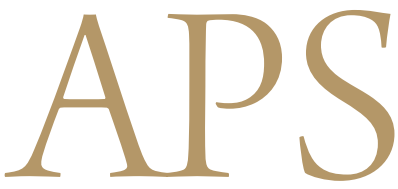

Archives of Plastic Surgery

\title{
The Influence of Pfannenstiel Incision Scarring on Deep Inferior Epigastric Perforator
}

\author{
Young Jin Park, Eun Key Kim, Ji Young Yun, Jin Sup Eom, Taik Jong Lee \\ Department of Plastic Surgery, Asan Medical Center, University of Ulsan College of Medicine, Seoul, Korea
}

Background Abdominal wall free flaps are used most frequently in autologous breast reconstruction, and these flaps require intact and robust deep inferior epigastric perforator (DIEP) vessels. Pfannenstiel incisions are often present during preoperative visits for breast reconstruction and could potentially signal compromised blood supply to the lower abdominal wall. In this study, we compared the number of DIEP vessels between patients with and without Pfannenstiel incisions undergoing autologous breast reconstruction.

Methods A retrospective review of medical records was performed for patients with (study) and without (control) Pfannelstiel incisions ( $\mathrm{n}=34$ for each group) between June 2010 and July 2013. In addition to patient demographics, number of caesarian sections, and outcomes of free flap reconstruction, abdominal wall vasculature was compared using the preoperative computed tomography angiographic data between the groups. For each patient, vessels measuring greater than $1 \mathrm{~mm}$ were counted and divided into four sections of the lower abdominal wall.

Results The mean number of perforator vessels was 10.6 in the study group and 11.4 in the control group, which was not statistically different $(P=0.575)$. Pfannenstiel incisions with history of repeat caesarian sections were not associated with decreased number of perforator vessels.

Conclusions Pfannenstiel scars are associated with neither a change in the number of DIEP vessels nor decreased viability of a free transverse rectus abdominis myocutaneous and DIEP flap. Lower abdominal free flaps based on DIEP vessels appear safe even in patients who have had multiple caesarian sections through Pfannenstiel incisions.

Keywords Cesarean section / Rectus abdominis / Perforator flap
Correspondence: Eun Key Kim Department of Plastic and Reconstructive Surgery, Asan Medical Center, University of Ulsan College of Medicine, 88 Olympic-ro 43-gil, Songpa-gu, Seoul 138-736, Korea Tel: +82-2-3010-3600 Fax: +82-2-476-7471

E-mail: nicekek@korea.com

No potential conflict of interest relevant to this article was reported.

Received: 16 Jan 2014 • Revised: 3 Mar 2014 • Accepted: 12 Mar 2014

pISSN: 2234-6163 • elSSN: 2234-6171 • http://dx.doi.org/10.5999/aps.2014.41.5.542 • Arch Plast Surg 2014;41:542-547

\section{INTRODUCTION}

In South Korea, post-mastectomy breast reconstructions have been on the rise, with increasing incidence of breast cancer [1]. This increase has coincided with a high caesarian section rate, which was reported to be $40 \%$ of all live births delivered by Cesarean section for the year 2000 [2].
Depending on the level, orientation, and depth of abdominal incisions, the inferior epigastric vessels and its perforators can be injured during abdominal wall incisions. If the vessels are severely compromised, the choice of abdominal wall free flaps should thoroughly be scrutinized during preoperative planning of post-mastectomy breast reconstructions. In the population of concern-women in 40s and 50s-Pfannenstiel incisions are not 
uncommon, and the question arises as to whether the presence of such scars hold clinical and physiological significance for each patient who presents for breast reconstruction.

Anatomically, the Pfannenstiel approach begins as a small lower abdominal transverse incision on the surface, However, the deep fasciocutaneous flap is elevated more superiorly than the skin incision would indicate, often up to the level of the umbilicus, to allow for sufficient exploration of the central pelvis [3-5]. The deep inferior epigastric perforator (DIEP) vessels could be transected where they cross the plane between the rectus abdominis muscle and anterior rectus sheath. Theoretically, such occurrence may influence the vasculature of the abdominal wall and this would increase concerns about flap loss and complications of DIEP flap for breast reconstruction.

For reasons stated above, the interval between Pfannenstiel incision and DIEP flap harvest has decreased because breast cancer is being detected sooner and caesarian sections are being performed later. While the presence of a Pfannenstiel scar has not been a contraindication against DIEP flaps at our institution, we have noticed this changing epidemiology behind women who undergo caesarian section and thereafter require autologous breast reconstruction.

The aim of this study was to investigate whether Pfannenstiel incisions were associated with decreased vascularity of the abdominal wall in patients undergoing autologous breast reconstructions. We evaluate whether the location and number of perforator vessels are affected by either the interval from Cesarean section to breast reconstruction or the number of repeat Pfannenstiel incisions.

\section{METHODS}

A single-institution retrospective case-control study was performed to identify all patients who underwent immediate breast reconstruction between June 2010 and July 2013. Inclusion criteria was made for all patients who received either a free transverse rectus abdominis myocutaneous (TRAM) or DIEP flap in the presence of a pre-existing Pfannenstiel incision for caesarian section. A separate control group included patients who had received comparative breast reconstructions (DIEP or TRAM) during the same period but without any abdominal scars. The review identified 34 patients with Pfannenstiel incision for the study group, and the control group of 34 patients was matched to the study group, based on variables including age, body mass index, and medical history.

Routine preoperative computed tomography (CT) angiogram scans and Doppler ultrasound were available for evaluation of perforator vessels in all subjects. CT angiograms were obtained using a 64-multidetector computed tomography scanner (Somatom Sensation 64, Siemens Medical Solutions, Erlangen, Germany) under the following parameter settings: $\mathrm{kVp} 100$, effective mAs 200 to 300, gantry rotation time 0.5 second, slice thickness $2 \mathrm{~mm}$, matrix $512 \times 512$, and a field of view covering the entire trunk. Intravenous nonionic contrast was used with a total volume of $120 \mathrm{~mL}$ with a concentration of $400 \mathrm{mg} \mathrm{I} / \mathrm{mL}$ (Iomeron, Bracco, AL TANA Pharma GmbH, Konstanz, Germany), injected at a rate of $4 \mathrm{~mL} / \mathrm{sec}$ through an 18-gauge antecubital catheter. The scan was delayed by 15 seconds, with each image obtained every 1.25 seconds. A vessel was identified as a perforator if the diameter could be measure at the limit of image resolution of CT angiogram, which was $1 \mathrm{~mm}$. Thus, all of the perforators were of $1 \mathrm{~mm}$ or greater in diameter, which was felt to be a clinically appropriate limit.

For each subject, the lower abdominal wall into 4 sections (left upper portion, left lower portion, right upper portion, right lower portion) to evaluate whether the presence of a Pfannenstiel incision was associated with decreased number of perforator vessels. Perforator vessels superior from the level of umbilicus were excluded from evaluation. The number and locations of perforator vessels was recorded for each patient in this manner, and were compared between the two groups (Fig. 1). Additionally, the number of perforators was compared between patients who had breast reconstruction within 5 years of Pfannnenstiel incision and those who had breast reconstruction more than 5 years after Pfannenstiel incision.

Data were analyzed using SPSS ver. 21.0 (IBM Corp., Armonk, NY, USA). The Mann-Whitney U test was used to compare the number of perforator vessels between the study and control groups, as well as for the subgroup analysis A P-value less than 0.05 was considered statistically significant.

\section{RESULTS}

The mean age of the study group was 42.27 years (range, 37-52 years). The mean number of Pfannenstiel incision was 1.6, with 19 patients having 2 Pfannenstiel incisions and 2 patients having 3 Pfannenstiel incisions (Table 1). The intervals between the last Pfannenstiel incision and free flap breast reconstruction ranged from 1 to 24 years. The mean follow-up period was 18.3 months after the reconstructive procedure in the study group vs. 17.4 months for the control group.

The mean number of perforator vessels was 10.6 (range, 4 to 17) for the study group and 11.41 in the control group. Despite the slightly higher mean for the control group, the difference in perforator number was not statistically significant $(\mathrm{P}=0.57)$. Interestingly, patients with three Pfannenstiel incisions $(\mathrm{n}=2)$ 
Fig. 1. A 45-year-old patient requiring breast reconstruction

(A) Preoperative photograph shows superficial changes to the left breast skin, which was diagnosed as micro-invasive ductal carcinoma. The abdominal skin fold hides Pfannenstiel incisions from Cesarean section performed in 1990 and 1993. (B) Postoperative photograph at 6 months shows reconstructed the left breast, abdominoplasty scar, and previous Pfannenstiel incision. Skin sparing mastectomy with free transverse rectus abdominis myocutaneous was performed. (C, D) Pre-reconstruction computed tomography angiograms identified 14 perforator vessels.
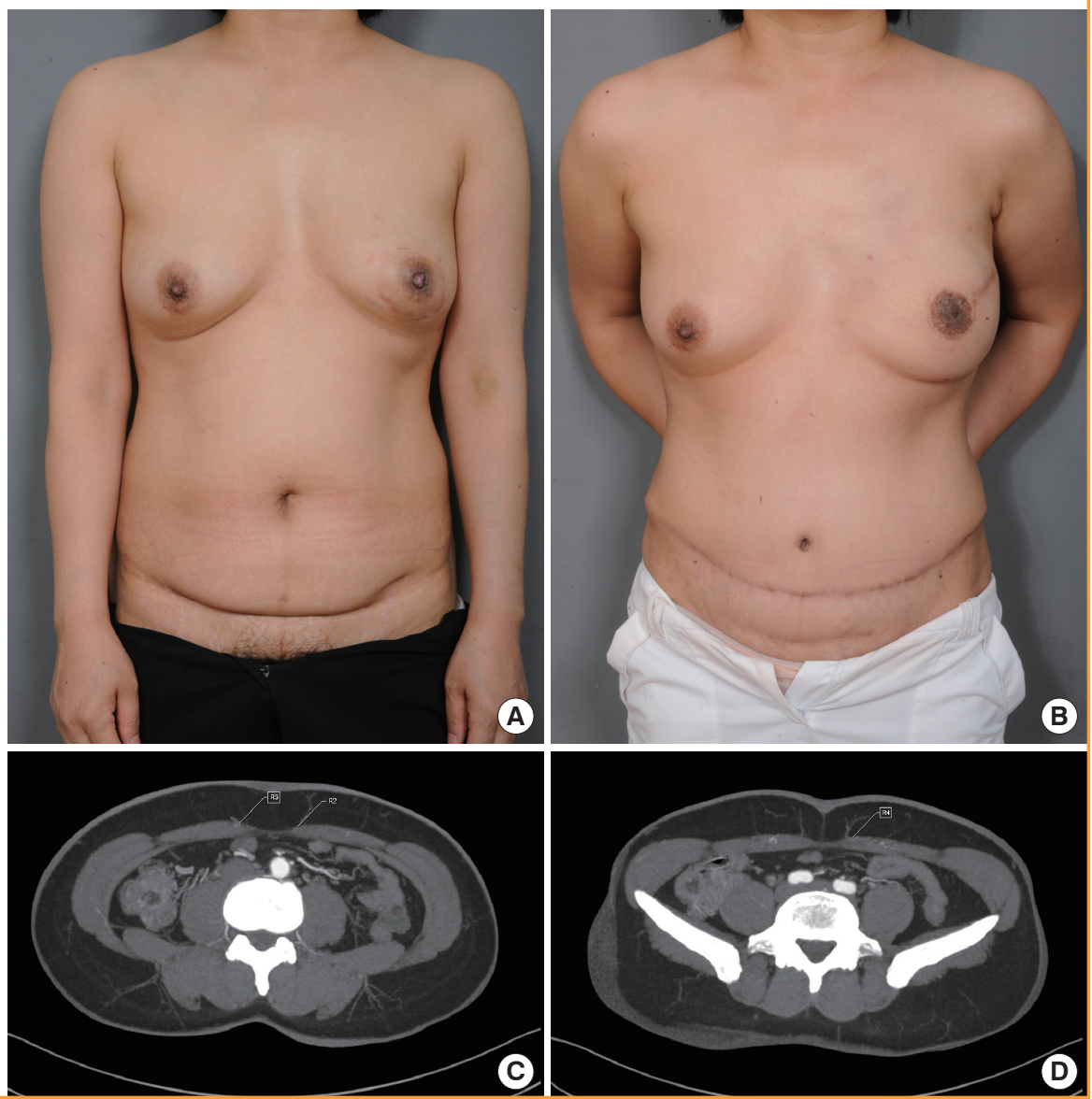

\section{Table 1. Patient demographics}

\begin{tabular}{|lcc|}
\hline Patients demographics & $\begin{array}{c}\text { Study group } \\
(\mathbf{n}=\mathbf{3 4})\end{array}$ & $\begin{array}{c}\text { Control group } \\
(\mathrm{n}=\mathbf{3 4})\end{array}$ \\
\hline Mean age $(\mathrm{yr})$ & 42.47 & 43.17 \\
Mean body mass index $\left(\mathrm{kg} / \mathrm{m}^{2}\right)$ & 24.22 & 23.15 \\
No. of Cesarean section & & \\
1 & 13 & 0 \\
2 & 19 & 0 \\
3 & 2 & 0 \\
Flap loss & 0 & 0 \\
\hline
\end{tabular}

were found to have 12 and 14 perforator vessels. The mean number of perforator vessels in lower-most abdominal locations was 1.73 in the study group and 2.09 in control group. No significant difference in distribution of perforator vessels was observed between the study group and control group $(\mathrm{P}>0.05)$ (Fig. 2). Subgroup analysis of the time intervals between Pfannelstiel incision and abdominal flap (less than 5 years vs. more than 5 years) did not identify any difference in perforator numbers $(\mathrm{P}=0.19)$ (Fig. 3). There were no flap losses in either the control or study groups.

\section{DISCUSSION}

Free TRAM and DIEP flap depend on the deep inferior epigastric perforating vessels from the rectus muscle and are regarded as the gold standard of autologous breast reconstruction. However, the use of a DIEP-based flap is somewhat controversial in the presence of an abdominal scars after a laparotomy or abdominoplasty. The presence of such abdominal scars may indicate that the perforator vessels feeding the anterior abdominal wall may have been compromised and is a cause for concern when considering abdominal free flaps in breast reconstructions. Hartrampf and Bennet [6] initially demonstrated that the TRAM flap procedure could be carried out safely with acceptable aesthetics and risk of complications in a selected patient population that excluded heavy smokers, morbid obesity, and patients with prior abdominal operations. Recent studies have reported contradictory findings regarding the relationship between previous abdominal incisions and TRAM flap complication rates. Watterson et al. [7] reported various risk factors for TRAM flap loss and donor site weakness, but considered the presence of Pfannenstiel scars "insignificant" enough to exclud- 
Fig. 2. Comparison of perforator vessels quantity according to location

The lower abdomen was divided into four areas. The mean number of perforator vessels was calculated for each of these areas. No significant differences were observed between study and control groups.

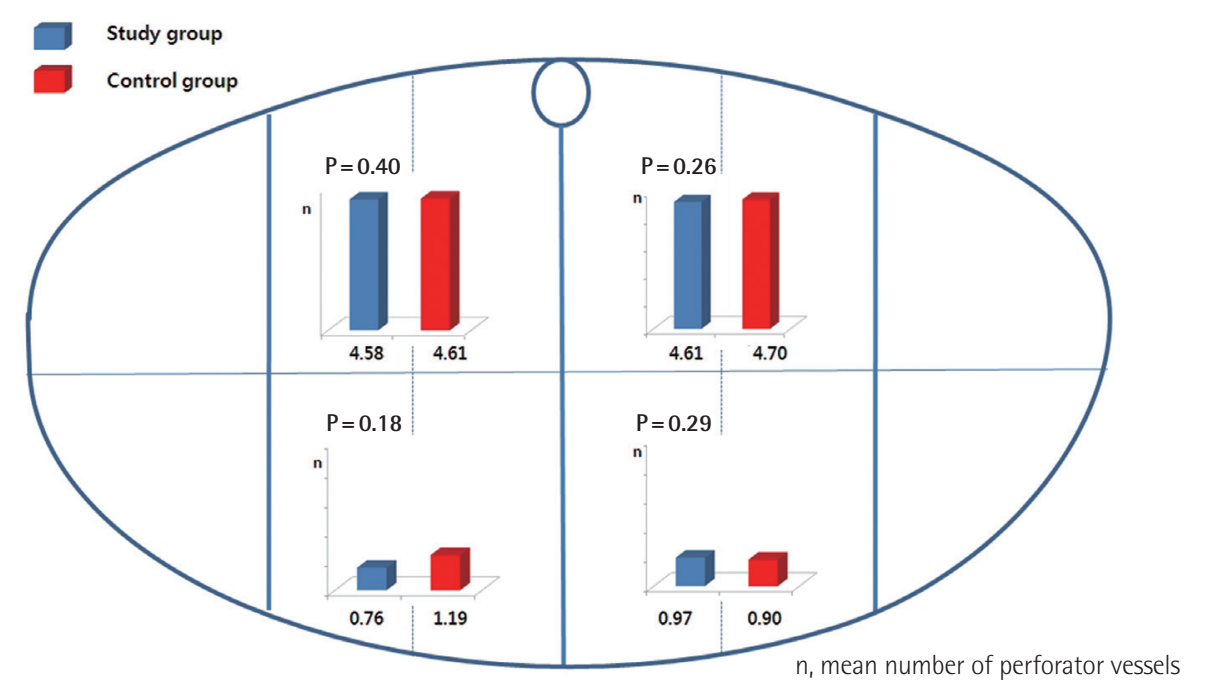

\section{Fig. 3. Perforator vessels according to time interval}

The number of perforator vessels did not correlate with the time interval between Cesarean section and breast reconstruction. Subgroup analysis did not identify any differences in perforator numbers between intervals less than five years and more than five years (Student t-test, $\mathrm{P}=0.19$ ).

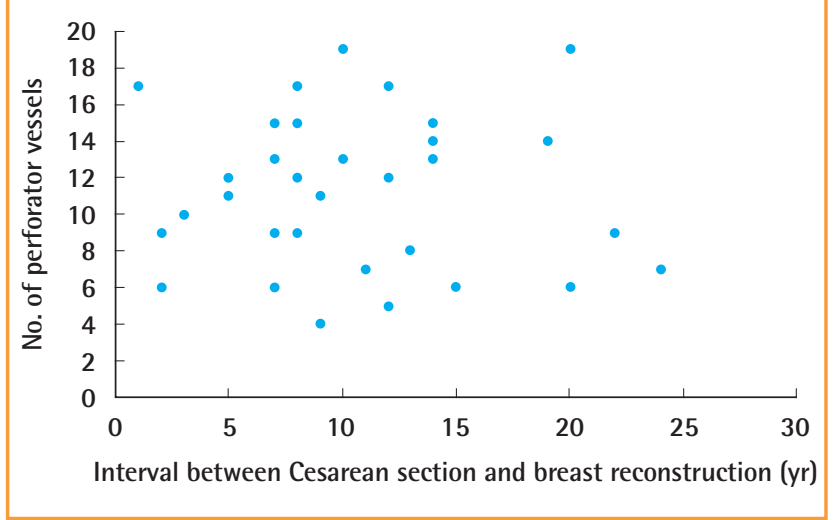

ed from analysis altogether. Additionally, Moon and Taylor [8] found no differences in the vascular pattern of anterior abdominal wall between cadavers with and without Pfannenstiel incision scars. On the other hand, Takeishi et al. [9] advocated a superior TRAM skin island design to avoid donor site necrosis in patients with low transverse abdominal scars.

In this retrospective study, we found that pre-existing Pfannenstiel scars were not associated with a significant difference in the number of perforator vessels from DIEA. Our finding as somewhat unexpected because the incision can transect the anterior rectus sheath perforators, which is the main source of occasional hematoma formation after Cesarean sections. While the harvest of free microsurgical TRAM flaps is considered safe even in patients with pre-existing abdominal scars [10], we anticipated that patients with Pfannenstiel scars would have a demonstrably lower number of perforator vessel compared to a control group. However, no such differences were found, which was a result consistent with findings by Mahajan et al. [11].

Abundance of perforator vessels after Pfannelstiel incision could potentially be explained by revascularization. In a study by Sozer et al. [12], prior abdominoplasty was reported not to interfere with successful TRAM reconstructions in two patients. The authors suggested that with sufficient time after abdominoplasty (10 years) adequate vascular architecture could have redeveloped. According to a report by Ribuffo et al. [13], the abdominal cutaneous vascular system was fully functional by one month after abdominoplasty, and the vessels continued to increase in size for up to six months. Mahajan et al. [11] also suggested an "ischemic pre-conditioning" component after partial disruption of the lower abdominal wall vascular network, by which smaller surviving perforator vessels grew in size to accommodate for the lost portion of circulation. In our comparison, we did not classify perforator vessels according to their diameters because $\mathrm{CT}$ angiography can only detect pulsatile perforator arteries with diameters greater than $1 \mathrm{~mm}$, which are considered adequately large enough for vascular anastomosis in autologous breast reconstructions. However, it may be possible that smaller perforator vessels not initially identified could become larger than $1 \mathrm{~mm}$ in caliber with time.

In addition to the possibility that smaller perforator vessels may grow in size after a trans-abdominal incision, the equivocal number of perforator vessels can also be explained by regeneration of transected perforator vessels.

Theoretically, the perforator vessels within the lower portion 
Table 2. Frequency of Cesarean section and number of perforator vessels

\begin{tabular}{|lcc|}
\hline $\begin{array}{l}\text { Frequency of } \\
\text { Cesarean section }\end{array}$ & Patients (no.) & $\begin{array}{c}\text { Mean perforator } \\
\text { vessels (no.) }\end{array}$ \\
\hline One & 13 & 9.92 \\
Two & 19 & 10.71 \\
Three & 2 & 13 \\
\hline
\end{tabular}

of abdomen should be more vulnerable during Cesarean section. Since abdominal location was not associated with perforator numbers, regeneration theory seems more plausible than the ischemic pre-conditioning theory.

With either of these mechanisms at work, increased time interval between Pfannenstiel incision and breast reconstruction should reveal higher numbers of perforator vessels. However, our subgroup analysis did not identify any differences in perforator numbers according to the time interval between Pfannenstiel incision and abdominal flap harvest. Likewise, no significant association was found between the number of Cesarean sections and number of perforator vessels (Table 2). In our clinical experience, we could find large perforator vessels during secondary abdominal flap elevation 3 years after TRAM flap breast reconstruction (Fig. 4). The revascularization may occur relatively early.

An unexplored but potentially confounding variable is the spectrum of technical variations in low transverse abdominal incisions among obstetric surgeons. In the present study, patients were presumed to have undergone Pfannenstiel incision from the location of external scar. However, the full dissection of abdominal wall may not have been of the classical Pfannenstiel type in which subfascial dissections are carried out as high as the level of umbilicus. However, some of the patients may have actually received Cherney or Maylard approaches, both of which preserve the rectus muscle perforators. Unfortunately, operative details regarding on the exact plane of dissection used for previous obstetric approaches were not available for the study cohort.

Through this case-control review, we have found that a pre-existing Pfannenstiel scar does not adversely impact the number of DIEP vessels and viability of a subsequent free TRAM or DIEP flap. This result supports previous investigations on the relationship between the obstetric incision and suitability of abdominal perforator vessels. While the physiologic basis for the commensurate number of perforator vessels between those with and without a Pfannenstiel incision is yet to be understood, the clinical implication of this phenomenon is that a Pfannenstiel does not contraindicate autologous breast reconstructions using abdominal free flaps in the growing number of women who present with a pre-existing Pfannenstiel scar.

\section{Fig. 4. Perforator vessels found 3 years after TRAM flap}

A 46-year-old patient had undergone transverse rectus abdominis myocutaneous (TRAM) flap breast reconstruction 3 years prior. The patient elected to undergo elective abdominoplasty for correction of soft tissue bulge. At the time of re-operation in the abdominal field, large perforator vessels were observed to be penetrating through the previous plane of dissection.

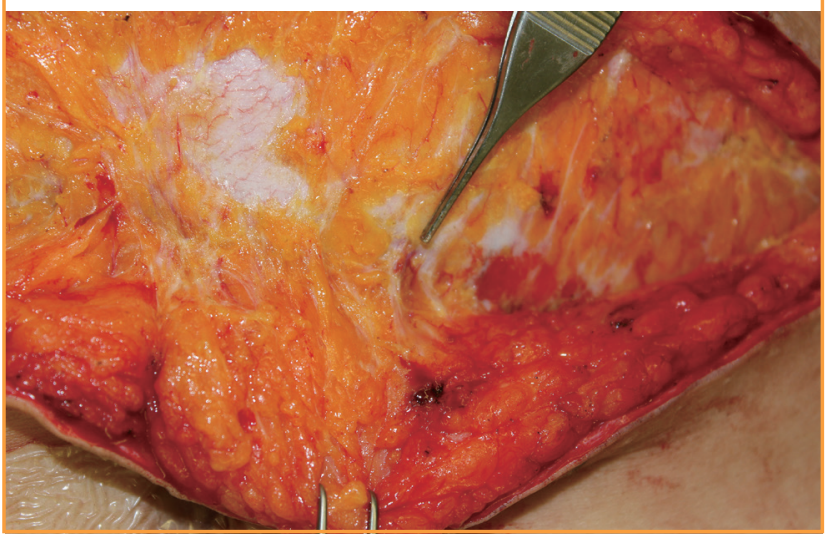

\section{REFERENCES}

1. Lee JH, Yim SH, Won YJ, et al. Population-based breast cancer statistics in Korea during 1993-2002: incidence, mortality, and survival. J Korean Med Sci 2007;22 Suppl:S11-6.

2. Lee SI, Khang YH, Lee MS. Women's attitudes toward mode of delivery in South Korea: a society with high cesarean section rates. Birth 2004;31:108-16.

3. Lees DH. Opening and closing the abdomen. In: Lees DH, Singer A, editors. Color atlas of gynecological surgery, Vol. 2. London: Year Book Medical Publishers; 1989. p.15-42.

4. Meeks GR. Clinical anatomy of incisions. In: Mann WJ, Stovall TG, editors. Gynecologic surgery. New York: Churchill Livingstone; 1996. p.121-68.

5. Nichols DH, Clarke-Pearson DL. Gynecologic, obstetric, and related surgery. St. Louis: Mosby; 2000.

6. Hartrampf CR Jr, Bennett GK. Autogenous tissue reconstruction in the mastectomy patient. A critical review of 300 patients. Ann Surg 1987;205:508-19.

7. Watterson PA, Bostwick J 3rd, Hester TR Jr, et al. TRAM flap anatomy correlated with a 10-year clinical experience with 556 patients. Plast Reconstr Surg 1995;95:1185-94.

8. Moon HK, Taylor GI. The vascular anatomy of rectus abdominis musculocutaneous flaps based on the deep superior epigastric system. Plast Reconstr Surg 1988;82:815-32.

9. Takeishi M, Shaw WW, Ahn CY, et al. TRAM flaps in patients with abdominal scars. Plast Reconstr Surg 1997;99: 713-22.

10. Schusterman MA, Kroll SS, Weldon ME. Immediate breast reconstruction: why the free TRAM over the conventional 
TRAM flap? Plast Reconstr Surg 1992;90:255-61.

11. Mahajan AL, Zeltzer A, Claes KE, et al. Are Pfannenstiel scars a boon or a curse for DIEP flap breast reconstructions? Plast Reconstr Surg 2012;129:797-805.

12. Sozer SO, Cronin ED, Biggs TM, et al. The Use of the
Transverse Rectus Abdominis Musculocutaneous Flap After Abdominoplasty. Annals of Plastic Surgery 1995;35.

13. Ribuffo D, Marcellino M, Barnett GR, et al. Breast reconstruction with abdominal flaps after abdominoplasties. Plast Reconstr Surg 2001;108:1604-8. 


\section{Discussion}

\section{The Influence of Pfannenstiel Incision Scarring on Deep Inferior Epigastric Perforator}

Lee Li-Qun Pu

Division of Plastic Surgery, University of California Davis, Sacramento, CA, USA

In this retrospective study, the authors evaluated whether a previous Pfannenstiel incision would change perforator flap anatomy based on the deep inferior epigastric perforator flap. They studied 34 patients from each group (Pfannenstiel incision group vs. control control) over a three-year period. Preoperatively computed tomography (CT) angiography was performed in each patient and the number of perforators $(>1 \mathrm{~mm})$ were counted and used as an end point of the study. They found that there was no statistical difference in the number of perforators in the Pfannenstiel incision group compared with the control group. The authors also found that the Pfannenstiel incisions with history of multiple caesarian sections were not associated with the decreased number of perforators in the deep inferior epigastric artery system. Both study groups have no flap loss and the reconstructive outcome is essentially the same from each group. Based on their study the authors have concluded that a Pfannenstiel scar is not associated with any changes in terms of number of perforators in the deep inferior epigastric artery system. An abdominally based deep inferior epigastric perforator (DIEP) flap appears to be safer even in patients who have had multiple Cesarean sections through pfannenstiel incisions [1].

Whether previous pfannenstiel incisions would change the number of perforators or anatomy of perforators based on the deep inferior epigastric vessels could be a critical decision preoperatively for this unique group of the patients. Obviously if a number of perforators or anatomy of perforators based on the deep inferior epigastric artery system has been altered from a previous Pfannenstiel Cesarean section, the perfusion to the flap based on these perforators can be compromised in a free DIEP flap or even a free transverse rectus abdominis myocutaneous (TRAM) flap. This can be especially true for Asian women since their body size are relatively small and a Pfannenstiel incision may, in theory, compromises to the perfusion of an abdominally based flap such as a free DIEP flap for a breast reconstruction.
Therefore, it is valuable to conduct such an important study to determine whether previous pfannenstiel incisions would have any impact on the perforators based on the deep inferior epigastric vessels in terms of the number of perforators or the anatomy of those perforators.

Before the surgeon performs an abdominally based breast reconstruction, especially a DIEP flap, it is critical he or she is able to find out more information about those perforators prior to the flap elevation [2]. It is true that the number of perforators or anatomy of perforator based on each deep inferior epigastric vessel system may not be the same. It is the surgeon's responsibility to identify the dominant side (left vs. right) before raising a DIEP flap if such a flap can be based on more number of perforators, larger perforators, as well as potentially less intramuscular dissection. For example, use either medial or lateral row perforators and there are two or three sizeable $(>1.5 \mathrm{~mm}$ with visible pulsation) on each row. Occasionally, the flap can be based on one large para-umbilical perforator $(>2.5 \mathrm{~mm}$ with visible pulsation). Therefore, knowing the detailed information about perforator anatomy would be critical to safely elevate a free DIEP flap. Besides knowing the size and location of the perforator, the flow-status of each selected perforator is much more important than simply the total number of perforators identified preoperatively by CT scan.

Although some surgeons prefer to use the preoperatively CT angiography to evaluate perforator anatomy prior to the breast reconstruction [3], I have found it is quite useful to use a $\mathrm{Du}$ plex scan to evaluate the perforators in the lower abdomen (Fig. 1) [4]. This type of study is often done in the operating room, prior to the skin incision. The surgeon could have direct interaction with the vascular technologist and in general the location of a number of important perforators can be identified [5]. In addition, the flow status of each perforator can be assessed. In this way the surgeon can decide which side of the DIEP flap can be raised. Obviously, if two or three large perforators can be identified in either medial or lateral row of the rectus abdominis muscle, a DIEP flap should be elevated in this selected side since more number and bigger size of the perforators with higher flow within this "dominant" side (Fig. 1). With knowing the perforator anatomy in such a detailed fashion, the surgeon can quickly and safely complete an elevation of a DIEP flap. Occasionally, a septocutaneous perforator can be identified and this would make the flap dissection relatively easy. However, there can still be some variations and incidental intra-operative findings during the flap dissection. The surgeon should have to prepare for 


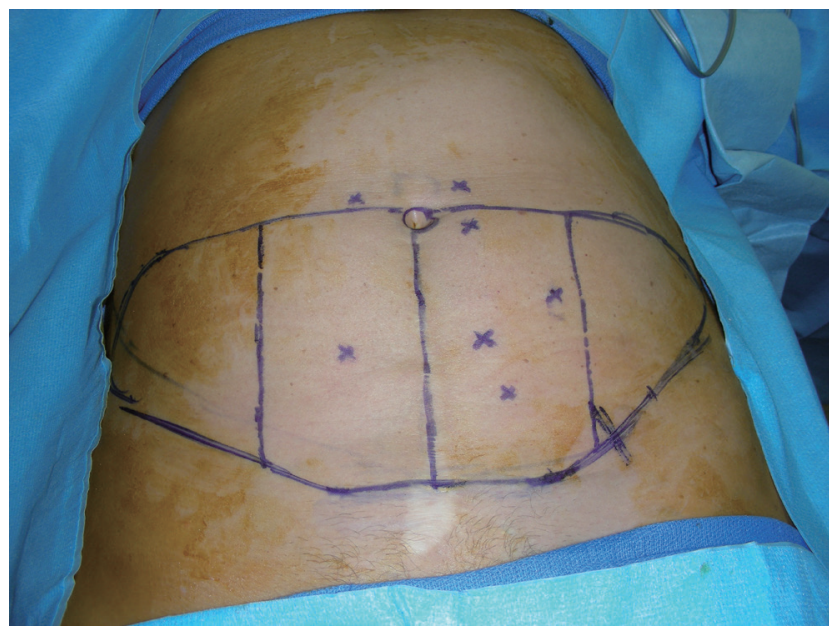

Fig. 1. An intraoperative view shows identified perforators from the patient's lower abdomen based on a color Duplex study. All perforators have been mapped but it is obvious that the deep inferior epigastric perforator flap should be chosen from the patient's left side because it is the "dominate" side with 2 or 3 larger perforators in the medial row. In this patient, the inferior epigastric vessels are also identified with the color Duplex study.

those anatomical variations of perforators and make a proper intra-operative judgment in terms of perforator dissection during an elevation of a free DIEP flap.

The authors should be congratulated to conduct such an important study. I believe the findings of this study can be another contribution to the plastic surgery literature because a number of perforators have not been changed significantly in the patients with a Pfannenstiel incision and elevation of a free DIEP or TRAM flap appears to be safe in this unique group of the patients at least based on this retrospective study. However, the conclusion based on the present study may be more meaningful if location of these perforators as well as the size or even the flow status of those perforators can also be evaluated. In addition, since the degree of tissue undermining for each Pfannenstiel incision can be quite different, the surgeon has to be cautious if pa- tient had a multiple previous Pfannenstiel incisions because occasionally the extent of surgical dissection might have been quite extensive and the inferior epigastric vessels could have been be transected. Any lower abdominal or groin incisions should be evaluated carefully since the inferior epigastric vessels could have been transected during an open inguinal hernia repair.

\section{REFERENCES}

1. Park YJ, Kim EK, Yun JY, et al. The influence of Pfanenstiel incision scarring on deep inferior epigastric perforator. Arch Plast Surg 2014;41:542-7.

2. Blondeel PN, Beyens G, Verhaeghe R, et al. Doppler flowmetry in the planning of perforator flaps. Br J Plast Surg 1998;51:202-9.

3. Teunis T, van Voss MR, Kon M, et al. CT-angiography prior to DIEP flap breast reconstruction: a systematic review and meta-analysis. Microsurgery 2013;33:496-502.

4. Rand RP, Cramer MM, Strandness DE Jr. Color-flow duplex scanning in the preoperative assessment of TRAM flap perforators: a report of 32 consecutive patients. Plast Reconstr Surg 1994;93:453-9.

5. Dorfman D, Pu LL. The value of color duplex imaging for planning and performing a free anterolateral thigh perforator flap. Ann Plast Surg 2014;72 Suppl 1:S6-8.

Correspondence: Lee Li-Qun Pu

Division of Plastic Surgery, University of California, Davis, 221 Stockton Boulevard, Suite 2123, Sacramento, CA 95817, USA

Tel: +1-916-734-2130, Fax: +1-916-734-7104,E-mail: lee.pu@ucdmc.ucdavis.edu

No potential conflict of interest relevant to this article was reported.

Received: 30 Apr 2014 • Revised: 30 Apr 2014 • Accepted: 30 Apr 2014 pISSN: 2234-6163・ elSSN: 2234-6171

http://dx.doi.org/10.5999/aps.2014.41.5.548 • Arch Plast Surg 2014;41:548-549 